\title{
Competency parfait (a common sense-based clinical practice guideline)
}

Competency assessment:

90-something-year-old gourmand de la vie.

10 years retired bush pilot, WWII vet, no family,

few remaining friends, endless stories,

fearless.

Stubborn heart.

Tired vessels.

Amputation.

Hospital: nursing home.

Patient: middle finger.

Two parts input from said patient.

Half part input from

fully qualified

licensed

well-intentioned

health care providers.

Simmer on low heat.

Stir in recent charting on file until dissolved.

Add clock drawing (prefer fresh, if available/in season).

Continue stirring until consistency thickens.

Layer into serving bowl and chill in refrigerator overnight.

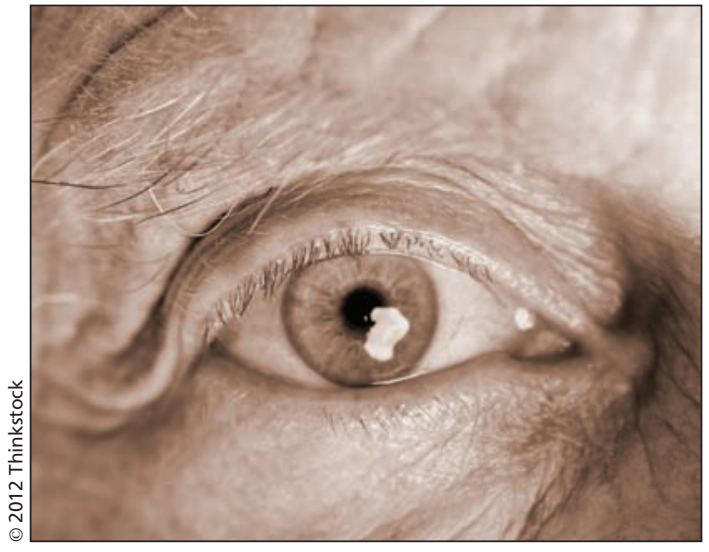

Portion and plate topped with a warm coulis of said patient's Gallic panache. Serve with a tall glass of cold-filtered indignation at the state of our system a.k.a.

the system of our state.

Michael J. Passmore MD

Geriatric Psychiatry

Clinical Assistant Professor

University of British Columbia

Vancouver, BC

CMAJ 2012. DOI:10.1503/cmaj.120339 